\title{
THE HEAT PUMP
}

\section{Possibilities in Energy Supply Systems}

The ability to transform heat into mechanical or other forms of energy of higher order has contributed to deep changes in social, economic, political and other aspects of human life over the past centuries to the point that almost everybody is now able to harness more (mechanical) horses to his vehicle than any Roman emperor. An inverse action, namely the use of mechanical energy for directing heat away from equilibrium is also already well known in refrigerating technology. The term "heat pump" is, however, usually applied only to processes and devices using heat from a natural or waste source and delivering it at a higher temperature for subsequent exploitation. This duty is almost identical with refrigeration except for the nature of the useful part and the temperature levels.

\section{Thermodynamics}

Let us consider two systems represented schematically in Fig. 1; one of them for mechanical power generation and the other for heat pumping. Each system is connected with one heat source and one heat sink. Let the temperatures at the system limits be constant (but different) at each of the above connections and let us designate the higher value of the temperature by $T_{1}$ the lower by $T_{2}$, the corresponding heat fluxes by $Q_{1}$ and $Q_{2}$ and the mechanical energy flux by $W$. Let us consider all fluxes leaving any of the systems as positive and the opposite fluxes as negative. The first and second law of thermodynamics might then be written in identical form for both systems:

$$
\begin{aligned}
& Q_{1}+Q_{2}+W=0 \\
& Q_{1} / T_{1}+Q_{2} / T_{2} \supseteq 0
\end{aligned}
$$

It is easy to extend the above relations to systems using any number of heat sources and heat sinks. In the particular case of a heat-driven heat pump, a second source of heat $Q_{0}$ at a temperature $T_{0}>T_{1}$ might be used instead of the mechanical energy $W$. The corresponding terms will then be added to the left hand sides of equations (1) and (2).

The equals sign in equation (2) is valid for idealized, reversible systems while the inequality in the indicated direction is significant for most real systems. The original situation cannot, therefore, be fully re-established for subsequent transformations of heat into mechanical energy and/or vice versa.

The ratio of the heat output $Q_{1}$ of a heat pump to the required mechanical energy $W$ or heat $Q_{0}$ at higher temperature is usually called the Coefficient Of Performance (COP) of the pump. Its maximum value is achieved, when the system is reversible, and so for a mechanically driven heat pump:

$$
\begin{aligned}
(\mathrm{COP})_{\max } & =-\left(Q_{1} / W\right)_{\max } \\
& =T_{1} /\left(T_{1}-T_{2}\right)
\end{aligned}
$$

and for a heat-driven heat pump:

$$
\begin{aligned}
& (\mathrm{COP})_{\max }=-\left(Q_{1} / Q_{0}\right)_{\max } \\
& =\left(1-T_{2} / T_{0}\right) /\left(1-T_{2} / T_{1}\right)
\end{aligned}
$$

For a given temperature level $T_{1}$, at which the heat $Q_{1}$ is required, the (COP) ${ }_{\text {max }}$ depends strongly on the temperature difference $T_{1}-T_{2}$. If heat could be delivered e.g. at $T_{1}=300 \mathrm{~K}$ for space heating and $a$ heat source is available at $T_{2}=280 \mathrm{~K}$, the $(\mathrm{COP})_{\max }$ of a mechanically driven heat pump could, theoretically be as high as 15. For the same conditions,

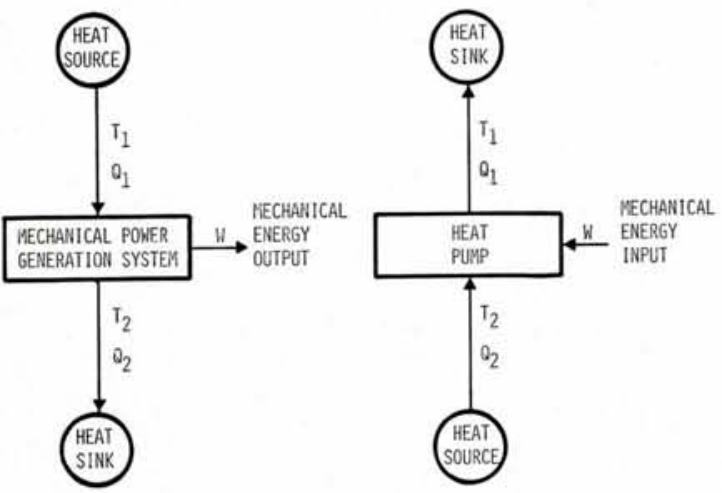

Fig. 1 Block diagram of a thermal power plant and a heat pump.

\section{Silberring, Zurich}

(Consulting Engineer)

the (COP) $)_{\max }$ of a heat pump driven by heat available, for example at $T_{0}=600 \mathrm{~K}$, would be eight; i.e., in the ideal case, for each unit of heating energy introduced at higher temperature $T_{0}$, seven additional units could be pumped from the lower temperature heat source at $T_{2}$ up to the required temperature level $T_{1}$. The actual value of COF' is lower than the above maximum value due to the non-equilibrium phenomena within the transformation system. The ration of the two values is a good measure of the quality of a heat pump and is often called efficiency $\eta$, for which the following relationship holds for a mechanically driven heat pump:

$$
\begin{aligned}
& \eta=\mathrm{COP} /(\mathrm{COP})_{\text {max }}=\left(Q_{1} / W\right) /\left(Q_{1} / W\right)_{\text {max }} \\
& =\left(W_{\min } / W_{Q_{1}}\right. \\
& =\left(1-T_{2} / T_{1}\right) /\left(1+Q_{2} / Q_{1}\right)
\end{aligned}
$$

and for a heat-driven heat pump:

$$
\begin{aligned}
\eta & =\mathrm{COP} /(\mathrm{COP})_{\max } \\
& =\left(Q_{1} / Q_{0}\right) /\left(Q_{1} / Q_{0}\right)_{\max } \\
& =\left(Q_{0} \min \right. \\
& \left.=\left(1-Q_{0}\right){ }_{Q_{1}} / T_{1}\right) \times\left(1-T_{2} / T_{0}\right) /\left(1+Q_{2} / Q_{1}\right)
\end{aligned}
$$

It will be noted that one of these values is negative according to the previously adopted definition. Thus $1+Q_{2} / Q_{1}=1-\left|Q_{2} / Q_{1}\right|$

The efficiencies, as defined above, do not depend significantly on the temperature level of the heat source or heat sink. The numerical values are between 0.2 and 0.8 for most known heat pumps, and close to the upper value of the above range for most power generating systems. This reflects the higher degree or development of the last mentioned systems as compared to the former.

The rather low efficiency of small heat pumps originates mainly in those requirements related to simplicity and reliability as they have to operate over prolonged periods without supervision and/or any substantial maintenance by skilled personnel. Furthermore, most devices on today's market have been developed for refrigerating duties at a time when low cost electrical energy was available. They are consequently not too suitable for heating duties in the present energy situation. Some improvements may be

cont. on page 9 
expected from a design better adapted to the actual requirements, but it cannot be anticipated that the efficiency of small heat pumps will approach unity. Some details about the essential origins of energy dissipation and related losses will be explained in the following text.

\section{Processes}

Most heat pump processes run using a fluid, circulating in a closed cycle of varying thermodynamic conditions. All relevant conditions can be described by two parameters, provided the fluid consists of a chemically stable single substance or of a mixture of constant composition. The cycle can then be represented graphically by a closed line.

The ideal cycle is the well known Carnot cycle provided the heat is available at one constant temperature and required at another constant temperature. The key advantage of the Rankine steam cycle for mechanical power generation is the relative small fraction of power, necessary to drive the cycle by the boiler feed pump, since the specific volume of the condensed state is many times less than the specific volume of stean. The same relationship between the specific volumes is advantageous for small heat pumps at least, as the pressure reducing part of the cycle can be completed without excessive entropy generation by a simple throttling device instead of an expansion machine The corresponding process is often called the Linde cycle.

Good heat exchange between evaporating liquid or condensing vapour and the surface of the corresponding exchangers represents another important advantage of vapour cycles for both power generation and heat pumps. Steam is seldom used as a working fluid for heat pumps due to its low pressure and high specific volume near ambient temperature. Fluids boiling at lower temperatures (or higher pressures) like ammonia or fluorinated hydrocarbons lead to a significantly smaller physical size of equipment for a given duty. Vapour cycles using saturated vapour only can provide a good approach to the Carnot cycle, but it should be remembered that this cycle is the best only for the particular case mentioned. In many practical cases, the sensible heat of fluids, the flow rates of which are limited, is used as heat source and/or heat sink. A Brayton gas cycle, familiar in power generation through gas turbines can provide a better fit for these last conditions. The same might be true for cycles using mixed vapours, whereas a number of more sophisticated cycles known in refrigerating and cryogenic technology could be appropriate for particular applications of heat pumps.

A heat-driven absorption heat pump process may use a mixture of at least two substances as a working fluid. Vapour and liquid of different compositions circulate by partially separate ways between a boiler heated by a source at an upper temperature and an absorber acting as a heat sink at the required temperature. Additional heat is delivered by a vapour condenser and picked up at lower temperature by evaporation of the condensed liquid at reduced pressure.

\section{Components}

Mechanically driven heat pump cycles contain at least four essential components (Fig. 2), namely a compressor, a throttling valve and two heat exchangers, one for heat withdrawal from the source and the other for heat delivery to the user. Any type of compressor can be used, provided the system is reasonably tight but hermetic or semi-hermetic compressors are preferred for small units, where the electric motor is sealed together with the compressor, and moving parts do not penetrate the containment of the working fluid. Larger units with external drives require shaft seals of more or less complicated design.

A typical mechanically driven heat pump uses as working fluid monochlorodifluoromethane ( $\left.\mathrm{CHCLF}_{2}\right)$, often called refrigerant 22 , evaporating at a pressure of 5.84 bar and a temperature of $5^{\circ} \mathrm{C}$, and condensing at a pressure of 19.4 bar and a temperature of $50^{\circ} \mathrm{C}$. The actual COP of this heat pump is between 3.6 and 5.0 , i.e. between $50 \%$ and $70 \%$ of $(\mathrm{COP})_{\max }=7.18$, depending on the unit size. Both evaporating and condensing temperatures and, consequently the COP, can vary widely depending on the external conditions, whereas the efficiency remains a characteristic of the device and varies only slightly.

Most heat pumps in existence operating on the Linde cycle are working some way away from the ideal conditions. Significant losses appear during compression, which cannot be completed isentropically and needs 20 to $30 \%$ more mechanical energy than the ideal process. Furthermore, evaporation, condensation and transport of fluids in pipelines are associated with pressure losses, which call for additional energy for com-

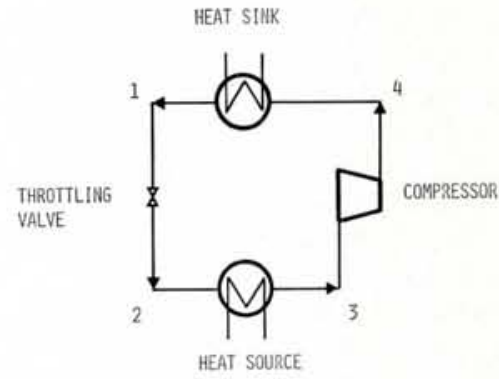

Fig. 2 Flow diagram of a mechanically driven heat pump.

pression. All this additional energy appears finally in the condenser, but the COP of its transformation is equal to unity and contributes therefore to the lowering of the overall efficiency. In hermetic and semi-hermetic compressors a greater than usual gap separates the motor rotor from the stator, leading to a lower motor efficiency, typically $85 \%$. Generally, the heat generated by the dissipation of the electrical energy is transmitted to the working fluid between the evaporator outlet and compressor inlet. Consequently, the vapour entering the compressor is superheated, and the energy required for compression rises again.

The efficiency can be increased by a number of measures, a few of which are described below. The cycle can be improved by a multistage compression with intermediate cooling and multistage throttling followed by recycle of flash vapour. Internal heat exchange between high and low pressure fluid may also be useful and the electric motors are of higher efficiency if Incated externally. Almost any improvement of efficiency calls for added complexity and cannot be economically justified below some minimum capacity of a single unit.

$A$ heat-driven absorption heat pump can be built using only heat exchangers and a liquid pump to circulate the fluids (Fig. 3), wich can even be omitted if the gravity potential difference between the absorber and boiler is adequate. In this case an absorption heat pump does not need any mechanical moving elements.

With aqueous ammonia as working fluid, the following conditions could be established in a heat-driven heat pump. Liquid solution, containing a $31 \%$ mass fraction of ammonia in water is fed into the generator, where it is separated at a pressure of 10 bar and a temperature of $145^{\circ} \mathrm{C}$, using external heating, into an ammoniarich vapour fraction, containing $59 \%$ ammonia, and an ammonia-lean liquid fraction, containing only $12 \%$ ammo- 
nia. The vapour solution is condensed at nearly the same pressure at a temperature of $50{ }^{\circ} \mathrm{C}$, then the condensate throttled down to a pressure of 2.5 bar and subsequently evaporated again at a temperature of $5^{\circ} \mathrm{C}$, picking up heat from the low temperature heat source. The low pressure vapour flows to the absorber, where it meets the ammonia-lean liquid solution from the generator. As a consequence, the vapour condenses again at a pressure of 2.5 bar and a temperature of $50{ }^{\circ} \mathrm{C}$, delivering the bulk of the heat introduced into the generator at higher temperature. The liquid solution from the absorber is returned via a pump to the generator. In this example, the heat delivery in the condenser occurs at the same temperature as in the absorber, namely at $50^{\circ} \mathrm{C}$, but in a more general case the temperatures would be different.

The heat delivered in the condenser is nearly equal to the additional heat picked up in the evaporator. The COP of the system is defined as the ratio of the heat delivered in the condenser and the absorber to the heat introduced into the generator. Its value is 1.7 , i.e. $71 \%$ of the $(C O P)_{\max }=2.4$.

Both COP and (COP) $)_{\max }$ are lower than in the case of mechanically driven heat pumps due to the lower grade of the driving energy. Higher values of COP could be achieved using working fluids suitable for a heat input at higher temperature, and while there are potential candidates, none so far has found a widespread use. The efficiency of the pump quoted is relatively high, but additional increase is still possible, e.g. by process optimising for heating rather than refrigerating purposes, by internal heat exchange between different streams of the working fluid and by the use of counter-current columns instead of vessels for mixing anci/or demixing liquid and gaseous solutions. Other processes are also possible. For example, the absorber might operate at higher pressure than the generator. In this case, one part of the heat, available at an intermediate temperature, is discharged at a lower temperature, while the remaining part is "pumped" to a higher temperature.

\section{Heat Sources and Sinks}

Every heat pump needs either a waste or a natural heat source. Ambient air is available nearly everywhere at no cost (except in holiday centres) and so far, neither the cooling nor the heating of ambient air is penalised. The same cannot be said about natu-

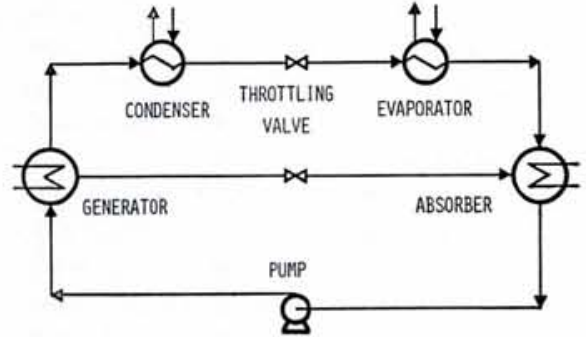

Fig. 3 Flow diagram of a heat-driven heat pump.

ral waters, which are subjected to a number of legal restrictions in most countries. Apart from cooling the water, there is the danger of water pollution by the working fluid and/or lubricating oil due to the leaks from evaporators of the heat pumps which can be kept very low (certainly lower than the danger of leaks from domestic oil tanks), but not zero. General rules governing these questions could assist local authorities in making decisions about the future use of natural water for heat pumps.

Heat rejected from electrical power plants represents a valid heat source for heat pumps located in their neighbourhood, even if one could argue that district heating by water withdrawn from the plant at the required temperature level represents a more direct and efficient way. However, it is not easy to adapt big nuclear power plants to highly variable heat withdrawal, whereas cooling water can be taken off without major disturbance. This water cannot, though be transported economically over long distances, since its heating capacity is limited and large volumes are required for a given duty. In addition, it would have to compete with any locally available natural water.

Solar energy collectors could assist other heat sources in an integrated heat pump system.

Ambient air is a much worse heat source than any natural water. The heat capacity of a unit volume of air is about 4000 times less than that of water and the film heat transfer coefficient for air moving along a solid surface is less by about two orders of magnitude. Air is generally colder than natural water, when the highest performance of the heat pump is required. Moreover, much more energy is necessary to transport enough air across the heat exchanger than with water. In brief, the total average consumption of mechanical or electrical energy for a given heat output at required temperature is much higher it ambient air is used instead of natural water as a heat source.

The nature of the heat sink depends, of course, on the ultimate use of the heat, and leads to a higher efficiency if the heat can be delivered to a liquid rather than to a gas.

In this article, considerations relating to the efficiency of heat pumps have been limited so far to the process temperatures of the heat pump cycles. However, some temperature differences are necessary for the heat transfer from the heat source to the working fluid, as well as from the latter to the heat sink, and in hermetic units, some lubricating oil circulates together with the working fluid and contributes to an increase in heat flux resistance. Usual values of temperature difference between the lowest temperature of the bulk of a heating fluid and the evaporing temperature of the working fluid, or between the condensing temperature of the working fluid and the highest temperature of the bulk of a heated fluid, optimised against the heating surface of the heat exchangers are between 2 and $6 \mathrm{~K}$ if liquids films are involved on both sides of the heating surface. If low pressure gas, e.g. ambient air, is the heating or heated fluid the usual range is between 10 and $20 \mathrm{~K}$.

Where it is the sensible heat of fluids that is used as a heat source or heat sink, then the temperature drop or increase adds further to the temperature differences indicated above, and while these additions might depend on the restrictions controlling the flow rate of the fluids they should be optimised against the power necessary for their transport.

A typical water-heated heat pump for space heating by water, might use natural water at an inlet temperature of $10^{\circ} \mathrm{C}$, cooled down to $8^{\circ} \mathrm{C}$, while the working fluid of the heat pump evaporates at $5^{\circ} \mathrm{C}$. At the heat sink, water is heated from a temperature of $40^{\circ} \mathrm{C}$ to $46^{\circ} \mathrm{C}$, giving a $(\mathrm{COP})_{\max }$ calculated for the inlet temperatures of both heating and heated fluids of 10.44 as compared with 7.18 for the internal heat pump cycle. The internal cycle efficiencies of $50 \%-70 \%$ drop now to $34 \%-48 \%$, when related to the inlet temperatures of the external fluids. This drop in efficiency is significantly greater if air is used as heating fluid.

Simplified information about process conditions and essential details about energy requirements for a typical small electrically driven heat pump are given in Fig. 4 and Table 1. Bigger heat pumps can perform much closer to the Carnot efficiency. But such pumps, which might be used for industrial purposes, for the heating of large buildings or even limited dis- 
tricts, must be carefully selected and optimised in the context of the whole related heating and energy supply system.

It can be seen from formulae (3) and (4) that the ratio of the absolute temperature at which heat is available from a natural or waste heat source to the absolute temperature at which heat is required represents one important criterion for an assessment of the economic potential of a heat pump application. This ratio should preferably be close to unity and in any case not less than about 0.7 .

\section{Applications}

Various industrial applications of heat pumps have been known for a long time, but, until recently, cheap fossil fuels were mainly used for heat generation, even when the heat was required at a low temperature level only. Most heat supplied to industrial processes must finally be rejected either to the ambient air or to natural water. At least a partial recycle of this heat by heat pumps can therefore contribute not only to fuel economy but also to a reduction of the thermal pollution of the environment. The recent sharp rise in the cost of fuels will certainly favour this action.

Distillation processes are amongst the best candidates for the wide industrial application of heat pumps, in particular, if the ratio of the condensing temperature to the reboiling temperature is, as required, close to unity. Demineralisation of liquids, e.g. sea water, or the concentration of dissolved solids are particular examples. Separation of isotopes, e.g. heavy water, by distillation belongs also to the field of possible applications, provided the vapour pressure drop in the distillation column is not too high.

Nevertheless, space heating in areas of moderate climate represents by far the most important potential use of heat pumps. A wide application of these devices depends, however, on other factors.

Space heating technologies and customs have been developed over a long period. In most cases, fossil fuels for more or less direct heating were available at a cost, which was many times lower than any energy of higher order, e.g. mechanical or electrical. Thus, most space heating installations could accept a high rate of entropy generation with high temperature differences at almost all stages of heat transfer between the adiabatic fuel combustion at about $2000^{\circ} \mathrm{C}$ and the required room temperature of about $20^{\circ} \mathrm{C}$. Heat pumps

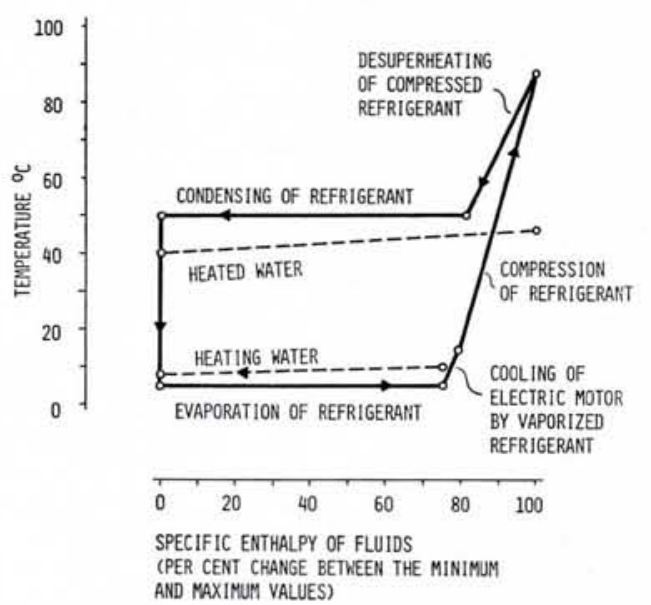

Fig. 4 Temperature-enthalpy diagram of a typical electrically driven (by canned rotor) water-heated and water heating pump. too need much energy of high order if they are to fit into some intermediate stage, although heating systems using moderate temperatures are now slowly being introduced and are essential for opening up the economic possibilities of heat pumps.

\section{Economic Considerations}

Air heated heat pumps can be fitted almost everywhere, but the seasonal average COP of installations using such pumps is only about 2 . As a consequence, the economic attractivness of these installations is limited. Heat pumps heated by natural water can achieve a seasonal average COP of about 4 .

In practice, economic policies will determine their rate of introduction. Electrical energy is, in most cases, sold to consumers on a basis similar to other goods and even though different prices may apply over different hours, the ratio of minimum to average value is rarely less than 0.5 . But as high capital cost generating stations become forced to operate on a diminishing load factor as the base load is taken up, the incentive for the supplier to widen the differential will increase. At the same time, the consumer, who in the past could do little to restrict his daily variations in demand, will be encouraged to take advantage of the lowered off-peak tariff by storing energy used for heating. Some electric storage heating systems already use off-peak energy; heat pumps can do the same, though much more economically, even if a greater volume of storage system is necessary.

It should be evident that heat pumps can make a significant contribution to a reduction in primary energy requirements. Facilities already exist for transporting and distributing the drive energy needed, so that heating can be provided by economic and almost pollution-free means.
TABLE 1 - Mechanical and electrical energy requirements of a typical electric canned motor driven water-heated and water heating heat pump for delivery of one unit of heat.

Mechanical energy requirement for evaporation at $10^{\circ} \mathrm{C}$ and condensation at $40^{\circ} \mathrm{C}$

\begin{tabular}{|c|c|}
\hline $\begin{array}{c}\text { Carnot } \\
\text { cycle }\end{array}$ & $\begin{array}{l}\text { Linde Cycle } \\
\text { using refrigerant } 2\end{array}$ \\
\hline & Ideal Real \\
\hline
\end{tabular}

Additional mechanical energy requirements :

Due to a temperature drop of the heating water by $2 \mathrm{~K}$ to $8^{\circ} \mathrm{C}$

Due to a temperature difference of $3 \mathrm{~K}$ between heating water at outlet and evaporation at $5^{\circ} \mathrm{C}$

Due to a temperature rise of the heated water by $6 \mathrm{~K}$ to $46^{\circ} \mathrm{C}$

Due to a temperature difference of $4 \mathrm{~K}$ between condensation at $50^{\circ} \mathrm{C}$ and heated water outlet

Total mechanical energy requirements Losses in electric motor of $85 \%$ efficiency

$\begin{array}{lll}0.0958 & 0.1115 & 0.1356\end{array}$

Additional electrical energy requirement

due to the cooling of the electric motor

by vapourized refrigerant

$\begin{array}{lll}0.0064 & 0.0080 \quad 0.0095\end{array}$

$\begin{array}{lll}0.0096 & 0.0123 & 0.0144\end{array}$

$\begin{array}{lll}0.0167 & 0.0243 & 0.0283\end{array}$

\begin{tabular}{lr}
0.0009 \\
\cline { 2 - 2 } Total electric energy requirement & 0.2445
\end{tabular} 